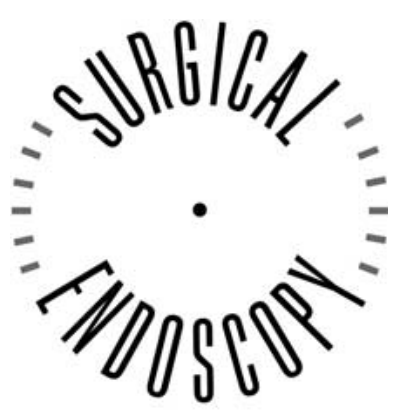

and Other Interventional Techniques

\title{
2004 Scientific Session of the Society of American Gastrointestinal and Endoscopic Surgeons (SAGES) Denver, Colorado, USA, 31 March-3 April 2004
}

\section{Oral presentations}

[Surg Endosc (2004) 18 (suppl): S157-S183, DOI: 10.1007/s00464-004-0067-4]

An abstract was mistakenly omitted from the Supplement to Volume 18. It should have appeared in the "Basic Science (Cellular Bio, Physiology)" section on pages S161-S162, and the abstract is printed here.

INFLUENCE OF $\mathrm{CO}_{2}$-, HELIUM- AND GASLESS PNEUMOPERITONEUM ON PORTAL, ARTERIAL AND CENTRAL VENOUS BLOODGASES IN AN EXPERIMENTAL MODEL

D. INDERBITZIN MD ${ }^{1,3}$, G. Marti', B. FELLAY PhD ${ }^{2}$, J.-L. MAGNIN PhD ${ }^{2}$, M. CELIO MD ${ }^{3}$, L. KRÄHENBÜHL $\mathrm{MD}^{1}$

1 Department of General and Visceral Surgery, Hôpital Cantonal, CH-1708 Fribourg, Switzerland

2 Department of Hematology and Clinical Chemistry, Hôpital Cantonal, CH-1708 Fribourg, Switzerland

INTRODUCTION: $\mathrm{CO}_{2}$-laparoscopy leads to acidosis and hypercapnia in peripheral arterial and venous blood (clinical studies). This may lead to postoperative tissue-damage and locally impaired immune-function. So far, portal venous blood-gases during laparoscopy are unknown. We assessed the influence of gas-quality and insufflation-pressure on portal, central-venous and peripheral arterial blood-gases during experimental laparoscopy. MATERIALS \& METHODS: 36 male rats were randomized into 6 groups $(n=6): 1$ median laparotomy (LT), 2-6 laparoscopy (LS) insufflating $\mathrm{CO}_{2}(6$ or $12 \mathrm{mmHg})$ or $\mathrm{He}(6$ or $12 \mathrm{mmHg})$ or gasless procedure. Animals were anaesthetized by isofluran/oxygen-inhalation and respiration-frequency kept constant at 40-45/min. Blood was sampled at time-points 45 and $90 \mathrm{~min}$ after setup from the portal and caval vein and femoral artery, transferred to hermetically closed glass-capillaries for blood gas analysis. Results are given as mean $\pm \mathrm{SD}$; stat. significance testing by ANOVA for repeated measurements and post-hoc comparisons by Bonferroni test. RESULTS: Intra-portal acidosis and hypercapnia were severe using $\mathrm{CO}_{2}$ (dependent on pressure) and high intra-abdominal pressure caused mechanical respiratory-impairment ( $\mathrm{He}$ $12 \mathrm{mmHg}$ ) Gasless LS and low-pressure He-insufflation allowed compensation of initial respiratory decrease. Arterial pH-decrease was no lower than $6.95 \pm 0.04\left(\mathrm{CO}_{2} 12 \mathrm{mmHg}\right)$. pO2 was not significantly affected $\left(\mathrm{O}_{2}-\right.$ inhalation). $\mathrm{HCO}_{3}$ displayed no significant compensatory effect. CONCLUSIONS: During $\mathrm{CO}_{2}$ pneumoperitoneum the liver is flushed by acidotic and hypercapnic portal blood which even affects the peripheral arterial blood-gases. This is mainly caused by direct intestinal $\mathrm{CO}_{2}$-uptake but also by mechanical respiratory-impairment due to high intra-abdominal pressure. Thus, low insufflation-pressures and using an alternative gas $(\mathrm{He})$ could ameliorate these effects. Combining low pressures and mechanical retractors would even lower the risk of-gas embolism.

\begin{tabular}{|l|c|c|c|c|c|c|}
\hline & \multicolumn{2}{|c|}{$0 \mathrm{~min}$} & \multicolumn{2}{c|}{$45 \mathrm{~min}$} & \multicolumn{2}{c|}{$90 \mathrm{~min}$} \\
\cline { 2 - 7 } & $\mathrm{pH}$ & $\mathrm{pCO}_{2} \mathrm{kPa}$ & $\mathrm{pH}$ & $\mathrm{pCO}_{2} \mathrm{kPa}$ & $\mathrm{pH}$ & $\mathrm{pCO} \mathrm{KPa}^{-}$ \\
\hline $\mathrm{LT}$ & $7.31 \pm 0.046$ & $7.14 \pm 0.89$ & $7.27 \pm 0.037$ & $7.82 \pm 0.93$ & $7.26 \pm 0.061$ & $7.29 \pm 1.42$ \\
\hline $\mathrm{LS} \mathrm{CO} 26 \mathrm{mmHg}$ & & & $6.99 \pm 0.065^{*}$ & $17.41 \pm 5.32^{*}$ & $6.94 \pm 0.036^{*}$ & $19.98 \pm 2.27^{*}$ \\
$\mathrm{LS} \mathrm{CO}_{2} 12 \mathrm{mmHg}$ & & & $6.87 \pm 0.043^{*}$ & $21.78 \pm 6.06^{*}$ & $6.83 \pm 0.056^{*}$ & $27.56 \pm 4.79^{*}$ \\
$\mathrm{LS} \mathrm{He} 6 \mathrm{mmHg}$ & & & $7.22 \pm 0.064$ & $9.65 \pm 2.03$ & $7.24 \pm 0.061$ & $8.53 \pm 2.57$ \\
$\mathrm{LS} \mathrm{He} 12 \mathrm{mmHg}$ & & & $7.17 \pm 0.025^{*}$ & $10.4 \pm 0.71$ & $7.14 \pm 0.045^{*}$ & $10.93 \pm 1.73$ \\
\hline $\mathrm{LS}$ gasless & & & $7.24 \pm 0.061$ & $9.25 \pm 1.52$ & $7.26 \pm 0.091$ & $8.26 \pm 2.47$ \\
\hline
\end{tabular}

Table: Intra-portal $\mathrm{pH}$ and $\mathrm{pCO}$ at 0,45 and 90 min after setup of $\mathrm{LS}$ and $\mathrm{LT}$ (mean $\pm \mathrm{SD}, \mathrm{p}<0.0005$ between operational groups, significant difference of $L S$ to $L T$ after Bonferroni correction $(p<0.005)$ ). 